\title{
RELAÇÕES ENTRE OS TIPOS E INDICADORES DE ACIDEZ DO SOLO EM LAVOURAS NO SISTEMA PLANTIO DIRETO NA REGIÃO DO PLANALTO DO RIO GRANDE DO SUL ${ }^{(1)}$
}

\author{
Margarete Nicolodi $^{(2)}$, Ibanor Anghinoni ${ }^{(3)} \&$ Clesio Gianello $^{(3)}$
}

\begin{abstract}
RESUMO
A recente mudança no sistema de cultivo do solo ocorrida no Brasil, passando do preparo convencional (PC) para o sistema plantio direto (SPD), altera o funcionamento do solo. No solo cultivado no SPD, as relações se estabelecem no tempo e são preservadas aumentando a complexidade do funcionamento do sistema, enquanto no PC estas relações são freqüentemente destruídas. Assim, devido à formação de gradientes de acidez, de matéria orgânica e de nutrientes a partir da superfície do solo é possível que se altere a magnitude das relações entre os tipos e os indicadores de acidez e que a camada de 0-20 cm de profundidade não seja a mais adequada para representar o estado de fertilidade de solo no SPD. Por essa razão, este trabalho foi conduzido com o objetivo de estudar as relações entre os tipos de acidez e os seus principais indicadores em duas camadas de solo em lavouras no SPD consolidado na região do Planalto Riograndense. Foram selecionadas seis lavouras, coletando-se, em cada uma, amostras de solo em 20 locais, nas camadas de 0-10 e 0-20 cm de profundidade, para a determinação dos tipos e dos indicadores de acidez. As relações entre os tipos e os indicadores de acidez do solo não se diferenciaram nas camadas avaliadas. Assim, para a região estudada e para o sistema de cultivo utilizado, qualquer das camadas (0-10 ou 0-20 cm) pode ser utilizada para fins de recomendação de calagem.
\end{abstract}

Termos de indexação: acidez potencial e ativa; alumínio; saturação por alumínio; saturação por bases.

\footnotetext{
(1) Parte da Dissertação de Mestrado da primeira autora, apresentada à Universidade Federal do Rio Grande do Sul - UFRGS. Trabalho realizado com apoio financeiro do CNPq e PRONEX. Recebido para publicação em maio de 2006 e aprovado em janeiro de 2008.

(2) Pós-Doutoranda em Ciência do Solo, Universidade Federal do Rio Grande do Sul - UFRGS. Av. Bento Gonçalves 7712, Caixa Postal 15100, CEP 90001-970 Porto Alegre (RS). E-mail: marganicolodi@hotmail.com

(3) Professor do Programa de Pós-Graduação em Ciência do Solo, UFRGS. E-mails: ibanghi@ufrgs.br; gianello@portoweb.com.br
} 


\title{
SUMMARY: RELATIONSHIPS AMONG ACIDITY TYPES AND INDICATORS IN SOILS UNDER NO-TILLAGE SYSTEM IN THE RIO GRANDE DO SUL PLANALTO REGION
}

\begin{abstract}
The recent shift in soil tillage from conventional to no-tillage systems in Brazil alters the soil system dynamics. In soils cultivated under the no-tillage system the relations are established and preserved over the course of time, which increases the functional complexity of the system, while under the conventional system often destroys these relationships. Some questions have risen about the reliability of sampling the so-called "arable soil layer" $(0-20 \mathrm{~cm})$ for soil acidity quantification in the no-tillage system due to the formation of acidity, organic matter and nutrient gradients in the top soil, besides modifications in the magnitude of the relationships between indicators and acidity types. To verify this type of relationships in no tillage systems, a study was conducted in six fields, in two layers $(0-10 \mathrm{~cm}$ and $0-20 \mathrm{~cm}$ ) of an Oxisol (Typic Hapludult) of the Planalto region in the state of Rio Grande do Sul. Representative soil samples were collected from 20 sites of each crop field and soil acidity was analyzed in the laboratory. The relationship between soil acidity types and indicators was not affected by the soil layer. For the no-tillage system in the region under study, both layers $(0-10 \mathrm{~cm}$ or $0-20 \mathrm{~cm})$ represent a reliable basis for lime recommendation.
\end{abstract}

Index terms: potential and active acidity; aluminum; aluminum saturation; base saturation.

\section{INTRODUÇÃO}

A recomendação de calagem é feita com base em indicadores de acidez do solo e na resposta das culturas à elevação de $\mathrm{pH}$. Os principais indicadores de acidez utilizados para a recomendação de calagem no Brasil são o $\mathrm{pH}$ em água, a saturação por bases, o $\mathrm{Al}$ trocável e, ou, Ca e Mg trocáveis (CFSRS/SC, 1995; Raij et al., 1996; Ribeiro et al., 1999; Wiethölter, 2000; Sousa \& Lobato, 2004). Essas recomendações foram desenvolvidas regionalmente para o preparo convencional (PC) de cultivo, com base na camada de solo de $0-20 \mathrm{~cm}$.

Para cada indicador de acidez, está associado um valor de referência (denominado critério de calagem) normalmente utilizado para um grupo de culturas. Assim, para o $\mathrm{pH}$ em água, o valor de referência é de 6,0 (CFSRS/SC, 1995) e para a saturação por bases é de $70 \%$ (Raij et al., 1996), para a maioria das plantas cultivadas. No entanto, é possível que tanto o indicador de acidez quanto o valor de referência não sejam adequados para recomendar calagem no solo cultivado por longo tempo no sistema plantio direto (SPD). Neste sistema, o revolvimento do solo é mínimo, com formação de gradientes a partir da superfície do solo, e a camada dita "arável" (0-20 cm) é substituída por outra enriquecida com resíduos orgânicos, alterando a dinâmica da acidez do solo, da matéria orgânica (MO) e da ciclagem de nutrientes (Sá, 1993; Bayer \& Mielniczuk, 1997; Anghinoni \& Salet, 1998).

Dentre as principais alterações na dinâmica da acidez em solos que acumulam MO, resultante da deposição de grande massa de resíduos e do baixo grau de decomposição desta, está a complexação do $\mathrm{Al}^{3+}$ por ligantes orgânicos, que diminui seu potencial químico na solução do solo (Salet, 1998; Miyazawa et al., 2000). Além disto, a diminuição do teor de $\mathrm{Al}$ trocável e de sua atividade na solução também decorre do acúmulo de P na superfície dos solos cultivados há tempos no SPD (Nolla \& Anghinoni, 2006). Diante dessa situação, torna-se necessário reavaliar as relações entre os indicadores de calagem, antes mencionados, entre si e a relação deles com os diferentes tipos de acidez (ativa e potencial, trocável e não-trocável).

Atualmente, altos rendimentos das culturas como soja, milho, trigo e cevada, dentre outras, são possíveis de ser obtidos em solos com $\mathrm{pH}$ baixo $(<5,5)$ e teor de Al trocável em nível considerado tóxico $(1,0$ $1,5 \mathrm{cmol}_{\mathrm{c}} \mathrm{dm}^{-3}$ ) para as plantas, quando cultivados há muitos anos no SPD (Anghinoni \& Salet, 1998; Caires et al., 1998; Fiorin et al., 1998; Pöttker \& Ben, 1998; Sá, 1999; Nicolodi et al., 2004). Uma possibilidade para o efeito diferenciado da acidez sobre as culturas é representada pela interação dos efeitos simples, com a formação de estruturas mais complexas que promovem a auto-organização do solo em nível de ordem mais elevado e melhora o ambiente para o crescimento e desenvolvimento das plantas (Mielniczuk, 2000; Vezzani, 2001). Nesse caso, os efeitos de interações seriam mais importantes que a soma dos efeitos simples e maior número de fatores influenciariam a produtividade das culturas com a participação proporcionalmente menor de cada um deles (D'Agostini, 2007).

A partir dessas considerações, surge a oportunidade do presente trabalho, com o objetivo de estudar as relações entre os tipos de acidez e seus principais indicadores em diferentes camadas de solo de lavouras cultivadas por longo tempo no sistema plantio direto na região do Planalto Riograndense. 


\section{MATERIAL E MÉTODOS}

Para o trabalho, foram selecionadas seis lavouras, cujo critério de escolha foi o de terem alta variabilidade nos indicadores de acidez (teor de Al trocável, saturação por $\mathrm{Al}$ e por bases e $\mathrm{pH}$ ) para bem representar as condições para as quais são elaboradas as recomendações de calagem. As lavouras estão localizadas em três municípios da região do Planalto do Rio Grande do Sul e, por ocasião da avaliação, já estavam no SPD há pelo menos cinco anos (SPD consolidado). A área das lavouras variou de 15 a 20 ha em Latossolo Vermelho distrófico. As lavouras 1, 2 e 3 localizam-se no município de Cruz Alta, a lavoura 4 no município de Não-Me-Toque e as lavouras 5 e 6 no município de Ibirubá, sendo cinco cultivadas com soja (Glycine max Merrill) e uma com cevada (Hordeum vulgare) e, posteriormente, com soja.

O histórico de cultivo das safras de verão 1998/ 1999 e de inverno de 2001 que antecederam a safra avaliada, o tempo de cultivo do solo no SPD, a amplitude e média dos rendimentos obtidos na safra avaliada, a adubação feita na cultura da soja (safra 2001/2002) e a última aplicação de calcário em cada lavoura são mostrados no quadro 1. Neste trabalho, os dados de rendimento são ilustrativos e não serão discutidos.

Foram avaliados 20 locais $(2,0 \times 2,4 \mathrm{~m})$ em cada lavoura, distribuídos de modo a contemplar a heterogeneidade do solo. Em cada local, foi coletada uma amostra de solo, composta por três subamostras, nas camadas de $0-10$ e $0-20 \mathrm{~cm}$ de profundidade. Cada subamostra de solo foi coletada com pá-de-corte e consistiu de uma fatia de $5 \mathrm{~cm}$ de espessura por $40 \mathrm{~cm}$ de largura, antes do cultivo da soja, e de $20 \mathrm{~cm}$, antes do cultivo da cevada, centralizada na linha de semeadura. Nas amostras de solo, foram determinados o $\mathrm{pH}$ em água, o índice SMP, os teores de $\mathrm{Ca}, \mathrm{Mg}$ e de Al trocáveis, MO, K e de $\mathrm{P}$ disponíveis (Mehlich 1), conforme Tedesco et al. (1995); o pH em $\mathrm{CaCl}_{2}$ 0,01 mol L-1, conforme Silva (1999); o valor da acidez potencial $(\mathrm{H}+\mathrm{Al})$ foi estimado pelo índice SMP, utilizando-se a equação de Kaminski et al. (2001), e a saturação por bases e por Al, por cálculo.

Neste trabalho, foram estabelecidas relações entre os tipos e indicadores de acidez do solo, utilizando, nas regressões, o modelo de melhor ajuste. Para isso foram feitas comparações entre os resultados pelo ajuste dos dados por regressão linear e não-linear, mesmo quando não houve relação de causa e efeito e foram colocadas as respectivas equações de ajuste com os coeficientes de correlação. Para as relações com causa e efeito, em vez do $R$, foi usado o coeficiente de determinação $\left(R^{2}\right)$. Os resultados foram apresentados desse modo para verificar o grau de associação e a linearidade entre as variáveis, razão por que as equações apresentadas são meramente ilustrativas.

Quadro 1. Tempo de cultivo do solo no sistema plantio direto, amplitude e média do rendimento de grãos de soja (safra 2001/02), última adubação e calagem e espécies cultivadas na rotação de culturas nas lavouras no Planalto do Rio Grande do Sul avaliadas neste trabalho

\begin{tabular}{|c|c|c|c|c|c|}
\hline \multirow{2}{*}{$\begin{array}{l}\text { Lavouras/ } \\
\text { município }\end{array}$} & \multirow{2}{*}{$\begin{array}{l}\text { Tempo } \\
\text { de SPD }\end{array}$} & \multicolumn{2}{|c|}{ Rendimento de grãos } & \multirow{2}{*}{$\begin{array}{c}\text { Adubação } \\
\left(\mathrm{N}-\mathrm{P}_{2} \mathrm{O}_{5}-\mathrm{K}_{2} \mathrm{O}\right)\end{array}$} & \multirow{2}{*}{$\begin{array}{c}\text { Última aplicação de } \\
\text { calcário }\end{array}$} \\
\hline & & Amplitude & Média & & \\
\hline & ano & $-\mathrm{t} \mathrm{ha}$ & - & $\mathrm{kg} \mathrm{ha}^{-1}$ & Ano $/ \mathrm{t} \mathrm{ha} \mathrm{h}^{-1} /$ modo \\
\hline 1. Cruz Alta & 5 & $1,8-6,0$ & 2,9 & $280(02-20-30)$ & 1998 / 4,0 / incorporado \\
\hline 2. Cruz Alta & 9 & $2,7-4,2$ & 2,5 & $250(00-20-30)$ & 1998 / 2,7 / superficial \\
\hline 3. Cruz Alta & 8 & $0,3-3,4$ & 2,1 & $280(00-12-30)$ & $1993 / 4,0 /$ incorporado \\
\hline 4. Não-Me-Toque & 6 & $2,3-4,4$ & 3,0 & $200(00-20-30)$ & $1995 / 3,0 /$ incorporado \\
\hline 5. Ibirubá & 5 & $2,8-4,2$ & 3,1 & $200(00-20-30)$ & $2000 / 2,5 /$ superficial \\
\hline 6. Ibirubá ${ }^{1}$ & 9 & $3,5-4,8$ & 4,0 & $200(00-20-30)$ & $1999 / 2,3 /$ superficial \\
\hline \multicolumn{6}{|c|}{ Espécies cultivadas na rotação de culturas do verão de 1998/99 ao inverno de 2001} \\
\hline 1. Cruz Alta & \multicolumn{5}{|c|}{ Soja / aveia / soja / trigo / soja / trigo } \\
\hline 2. Cruz Alta & \multicolumn{5}{|c|}{ Milho / trigo / soja / aveia + azevém / soja / aveia + azevém } \\
\hline 3. Cruz Alta & \multicolumn{5}{|c|}{ Milho / aveia + azevém / soja / aveia + azevém / soja / aveia + azevém } \\
\hline 4. Não-Me-Toque & \multicolumn{5}{|c|}{ Milho / cevada / soja / trigo / soja / trigo } \\
\hline 5. Ibirubá & \multicolumn{5}{|c|}{ Soja / trigo / soja / cevada / soja / trigo } \\
\hline 6. Ibirubá & \multicolumn{5}{|c|}{ Soja / trigo / soja / trigo / soja / cevada } \\
\hline
\end{tabular}

(1) Amplitude de rendimento de grãos de cevada: 2,1 - 3,0 t ha ${ }^{-1}$ (safra de inverno 2001). 


\section{RESULTADOS E DISCUSSÃO}

Os solos apresentaram, dentro de cada lavoura e no conjunto delas, grande amplitude de acidez e de tempo de cultivo no SPD (Quadros 1 e 2). Embora os solos de todas as lavouras sejam classificados como Latossolo Vermelho distrófico, os das lavouras localizadas em Cruz Alta (lavouras 1, 2 e 3) são mais ácidos e apresentam menores teores de argila (amplitude de 38 a $46 \%$ ) do que os das lavouras de Não-Me-Toque (lavoura 4) e de Ibirubá (lavouras 5 e 6) (amplitude de 58 a $75 \%$ ). A maior acidez observada nas lavouras de Cruz Alta pode ser explicada, em parte, pelo histórico de cultivo da região estudada (Quadro 2). A expansão das lavouras, em substituição à pecuária tradicional sobre campo natural, é relativamente recente, especialmente a partir da década de 90, enquanto, na região de Ibirubá e Não-Me-Toque, a agricultura já era praticada desde a década de $60 \mathrm{em}$ lavouras, tendo, a partir do início década de 90, ocorrido a mudança no sistema de cultivo, passando do PC para o SPD. Nestas lavouras (4 a 6), os solos tornaram-se menos ácidos e mais férteis com o tempo de cultivo. Também diferem de quando eram cultivados no PC, pois à época, embora houvesse aplicação de corretivos e fertilizantes, ocorriam grandes perdas de solo e de nutrientes devido à erosão. Assim, os solos desses municípios, cujo teor de MO e $\mathrm{H}+\mathrm{Al}$ são maiores do que os de Cruz Alta, tornaramse, no decorrer dos cultivos, menos ácidos e com maior fertilidade.

A dispersão dos pontos nas relações entre os tipos e os indicadores de acidez do solo neste trabalho (Figuras 1 a 5) é maior do que a observada em resultados de experimentos. Essa dispersão, tanto pelo número de pontos quanto pela natureza da avaliação, representa a diversidade dos fatores de solo e de planta que influenciam o rendimento das culturas. Mesmo assim, os valores de $\mathrm{R}$ e de $\mathrm{R}^{2}$ entre os tipos e os indicadores de acidez obtidos neste trabalho foram altos e muito significativos $(p<0,01)$.

\section{Relações entre acidez potencial e indicadores de acidez do solo}

$\mathrm{Na}$ avaliação conjunta dos pontos verifica-se (Figura 1a) que com o aumento da $\mathrm{H}+\mathrm{Al}$, ocorre diminuição proporcional do $\mathrm{pH}$ em água, mas de forma não-linear. A curva média praticamente separa os pontos em dois grupos distintos. Os solos mais argilosos, com maior teor de MO (Ibirubá e Não-MeToque), situam-se na parte superior e os menos argilosos, com menor teor de MO (lavouras da região de Cruz Alta), na parte inferior da curva traçada. Essa distribuição de pontos é atribuída, em parte, ao maior teor de $\mathrm{Al}^{3+}$ nos solos menos argilosos das lavouras da região de Cruz Alta. Como esses solos passaram a ser cultivados apenas recentemente, esses solos receberam quantidade menor de calcário no tempo.

No presente trabalho, mesmo que, eventualmente, a análise dos pontos em dois conjuntos proporcione um grau de associação um pouco maior entre as variáveis, a avaliação será efetuada para todo o conjunto de pontos, por sua maior representatividade da dispersão que naturalmente ocorre em condições de lavoura, para as quais são elaboradas as recomendações de calagem.

Na figura 1b, observa-se uma distribuição de pontos semelhante aos da figura 1a. Há, contudo, um deslocamento médio de 0,3 unidade de $\mathrm{pH}$, por se tratar de $\mathrm{pH}$ em $\mathrm{CaCl}_{2}$. Uma relação estreita entre a saturação por bases e a $\mathrm{H}+\mathrm{Al}$, porém de ordem inversa, pode ser observada na figura 1c. Também, nesse caso, em virtude da menor utilização de corretivos de acidez, os valores de Ca e Mg trocáveis são menores nos solos das lavouras da região de Cruz Alta. Com isso, a saturação por bases é, em média, menor para esses solos.

Quadro 2. Amplitude das características do solo avaliadas nas camadas de 0-20 e 0-10 cm de profundidade nas seis lavouras no sistema plantio direto consolidado na região do Planalto do Rio Grande do Sul

\begin{tabular}{|c|c|c|c|c|c|c|c|c|c|c|c|c|c|c|}
\hline \multirow{2}{*}{ 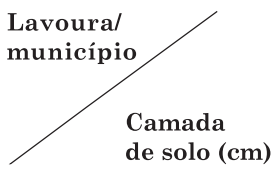 } & \multirow{2}{*}{$\begin{array}{c}\text { Argila } \\
0-20\end{array}$} & \multicolumn{2}{|c|}{ pH em água } & \multicolumn{2}{|c|}{ Al trocável } & \multicolumn{2}{|c|}{$\begin{array}{c}\text { Saturação } \\
\text { por Al }\end{array}$} & \multicolumn{2}{|c|}{$\begin{array}{c}\text { Saturação } \\
\text { por bases }\end{array}$} & \multirow[t]{2}{*}{$\mathbf{P}$} & \multirow{2}{*}{$\mathbf{K}$} & \multirow{2}{*}{$\mathrm{Ca}$} & \multirow{2}{*}{$\mathrm{Mg}$} & \multirow{2}{*}{ MO } \\
\hline & & $0-20$ & 0-10 & $0-20$ & $0-10$ & $0-20$ & 0-10 & $0-20$ & $0-10$ & & & & & \\
\hline & \multicolumn{3}{|l|}{$\%$} & \multicolumn{2}{|c|}{$-\mathrm{mmol}_{\mathrm{c}} \mathrm{dm}^{-3}-$} & 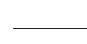 & — $\%$ & . & ـ & \multicolumn{2}{|c|}{$-\mathrm{mg} \mathrm{dm}^{-3}$} & \multicolumn{2}{|c|}{$-\mathrm{cmol}_{\mathrm{c}} \mathrm{dm}^{-3}-$} & $\%$ \\
\hline 1. Cruz Alta & $34-46$ & $4,4-5,8$ & $4,4-5,4$ & $1-17$ & $1-11$ & $1,2-50$ & $1,0-39$ & $19-72$ & $24-75$ & $5,1-30,4$ & $67-204$ & $1,6-4,1$ & $0,7-1,8$ & $2,0-3,4$ \\
\hline 2. Cruz Alta & $34-35$ & $4,8-5,7$ & $4,8-5,6$ & $1-8$ & $1-11$ & $0,8-16$ & $1,0-24$ & $38-68$ & $40-68$ & $3,0-15,6$ & $50-176$ & $2,9-3,4$ & $1,4-1,6$ & $2,1-2,9$ \\
\hline 3. Cruz Alta & $40-44$ & $4,3-5,2$ & $4,3-5,2$ & $1-22$ & $2-20$ & $1,9-45$ & $2,0-45$ & $20-64$ & $19-63$ & $2,3-28,0$ & $13-156$ & $3,1-3,8$ & $1,5-1,6$ & $2,4-2,7$ \\
\hline 4. Não-Me-Toque & $70-75$ & $5,0-6,2$ & $5,0-6,2$ & $0-4$ & $0-3$ & $0,3-4$ & $0,2-3$ & $54-80$ & $57-81$ & $6,7-36,6$ & $35-290$ & $6,5-8,2$ & $2,2-3,1$ & $3,6-3,9$ \\
\hline 5. Ibirubá & $58-65$ & $4,9-6,2$ & $5,1-6,2$ & $0-9$ & $0-6$ & $0,4-12$ & $0,1-1,9$ & $47-82$ & $62-85$ & $8,2-32,3$ & $65-158$ & $5,4-7,2$ & $2,4-3,4$ & $2,5-3,4$ \\
\hline 6. Ibirubá & $62-64$ & $4,7-6,5$ & $4,9-6,6$ & $0-7$ & $0-2$ & $0,2-14$ & $0,0-1,3$ & $43-88$ & $50-91$ & $3,2-25,7$ & $55-231$ & $7,2-8,8$ & $3,5-4,3$ & $3,3-3,8$ \\
\hline
\end{tabular}



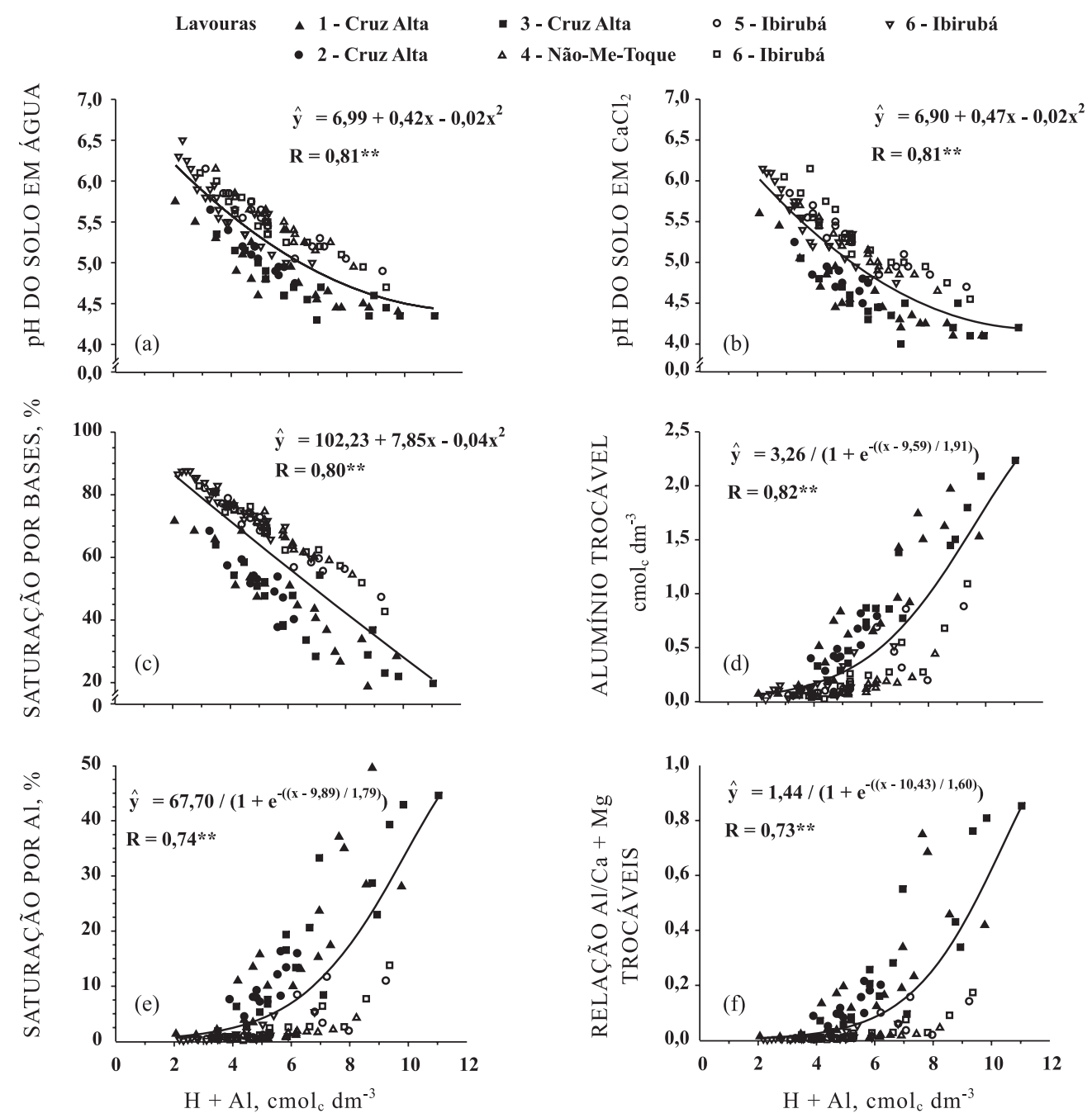

Figura 1. Relação entre a acidez potencial e os indicadores de acidez do solo: (a) pH em água; (b) pH em $\mathrm{CaCl}_{2}$; (c) saturação por bases; d) alumínio trocável; (e) saturação por alumínio; (f) relação Al/Ca + Mg trocáveis, em amostras coletadas na camada de 0-20 cm de profundidade, em lavouras no SPD.

Na figura 1d, 1e e 1f, observa-se relação não-linear entre o $\mathrm{Al}^{3+}$, ou relação deste com os outros cátions e a $\mathrm{H}+\mathrm{Al}$ do solo. Na maioria dos resultados obtidos nas amostras de solo da região de Ibirubá e Não-Me-Toque, a saturação por $\mathrm{Al}$ varia de baixa a muito baixa. Conseqüentemente, a distribuição dos pontos invertese em relação aos das figura 1a, 1b e 1c. Observa-se que, nesses solos, a saturação por $\mathrm{Al}$, em geral, é menor que $3 \%$ quando o teor de $\mathrm{H}+\mathrm{Al}$ é menor do que $6,0 \mathrm{cmol}_{\mathrm{c}} \mathrm{dm}^{-3}$ e aumenta acima deste valor, concomitantemente ao decréscimo do $\mathrm{pH}$ para valores menores que 5,5. Isso ocorre pelo $\mathrm{pH}$ abaixo de 5,5. Esse efeito manifesta-se em maior intensidade na camada de $0-20 \mathrm{~cm}$, uma vez que a calagem é feita na superfície do solo.

As relações entre a $\mathrm{H}+\mathrm{Al}$ e os três principais indicadores determinados na camada de $0-20 \mathrm{~cm}$ (Figura 1) para recomendação de calagem $(\mathrm{pH}$, saturação por bases e Al trocável), de modo geral, foram muito semelhantes, porém com os valores de $\mathrm{R}^{2} \mathrm{um}$ pouco maiores na camada de 0-10 cm (Figura 2). Contudo, como essa diferença é muito pequena, podese concluir que qualquer uma das camadas de solo (0-10 ou 0-20 cm) é adequada para recomendar calagem no SPD.

\section{Relações entre indicadores de acidez do solo}

Os valores de $\mathrm{R}$ entre os indicadores de acidez do solo (Figuras 3 a 5) foram altos $(R \geq 0,86)$ e muito significativos $(p<0,01)$, independente-mente da camada de solo utilizada. Verifica-se relação muito estreita entre o $\mathrm{pH}$ em água e a saturação por bases $(\mathrm{R}=0,96$ - Figura 3a e 3b), que não se alterou quando o pH foi determinado em solução de $\mathrm{CaCl}_{2}$ (Figura 3c e 3d) nas duas camadas de solo avaliadas.

A alta correlação entre o $\mathrm{pH}$ e a saturação por bases já era esperada, por ser esta um indicador indireto da acidez do solo. Com a neutralização do $\mathrm{Al}$ e do $\mathrm{H}$ pela 

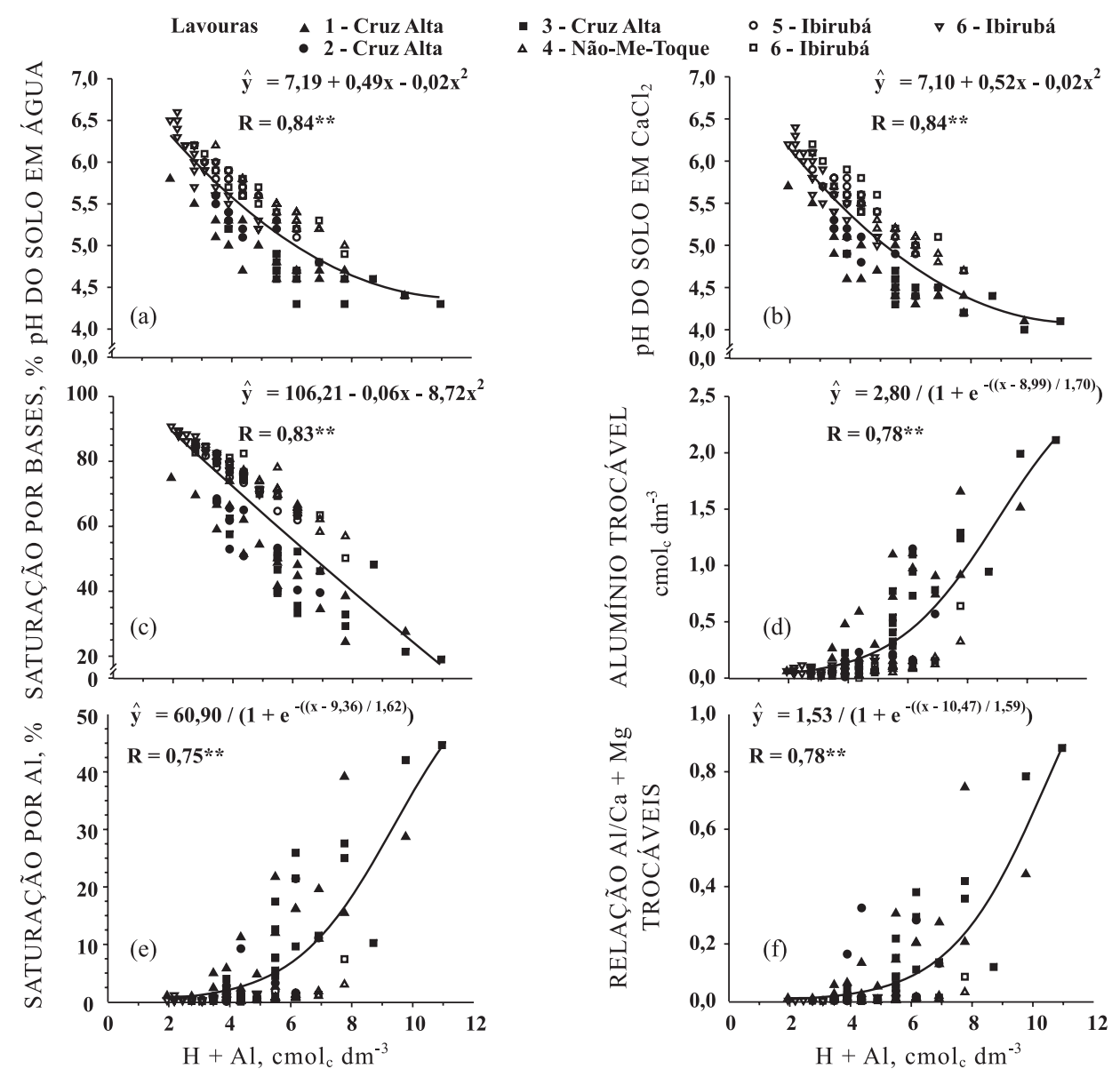

Figura 2. Relação entre a acidez potencial e os indicadores de acidez do solo: (a) pH em água; (b) pH em $\mathrm{CaCl}_{2}$; (c) saturação por bases; (d) alumínio trocável; (e) saturação por alumínio; (f) relação $\mathrm{Al} / \mathrm{Ca}+\mathrm{Mg}$ trocáveis, em amostras coletadas na camada de 0-10 cm de profundidade, em lavouras no SPD.

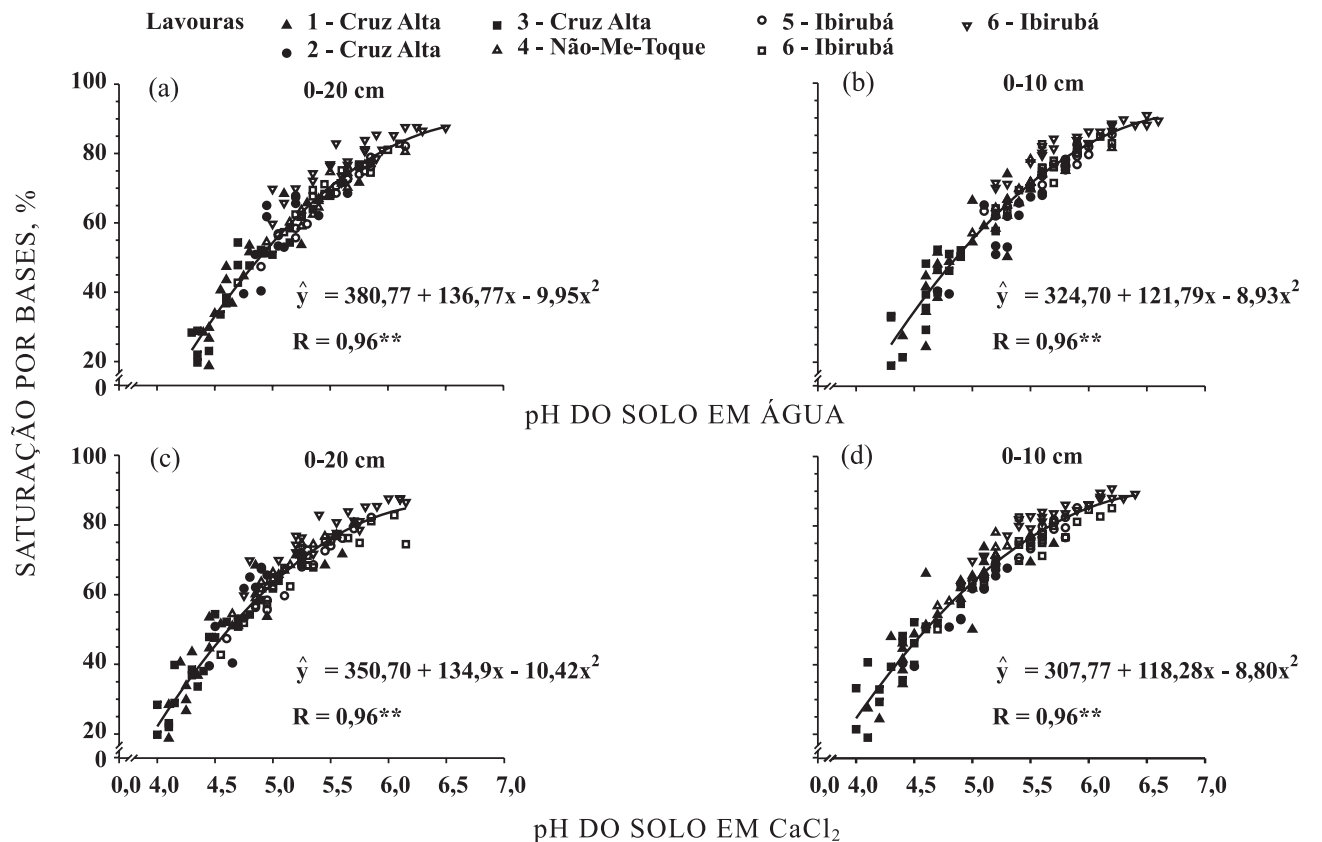

Figura 3. Relação entre o pH do solo determinado em água e em $\mathrm{CaCl}_{2} 10 \mathrm{~mm} \mathrm{~L} \mathrm{~L}^{-1}$ e a saturação por bases, em amostras coletadas nas camadas de 0-10 e 0-20 cm de profundidade, em lavouras no SPD. 
calagem, os sítios de troca catiônica são ocupados por bases trocáveis, aumentando, simultaneamente, o $\mathrm{pH}$ do solo e a saturação por bases (Cattani \& Gallo, 1955; Kaminski, 1974; Raij, 1989; Caires, 2000; Quaggio, 2000; Carballo, 2004). No presente trabalho e no de Carballo (2004), ambos no SPD, as relações obtidas entre esses indicadores foram não-lineares, ao contrário das relações lineares obtidas por Quaggio (2000) entre os mesmos indicadores em solos cultivado no PC em São Paulo, na camada de 0-20 cm. Souza et al. (1989) também obtiveram uma relação linear nessa camada com solos da região do Cerrado, porém com saturação por bases inferior a $35 \%$ para valores de $\mathrm{pH}$ de 5,5. À medida que os valores de saturação por bases aproximam-se de 80 a 90 \%, o ajuste da relação tornase não-linear.
O valor de pH 6,0 em água na camada de 0-20 cm, critério adotado pela CFSRS/SC (1995) para recomendação de calagem no solo cultivado no PC para os Estados do Rio Grande do Sul e de Santa Catarina, corresponde a $82 \%$ de saturação por bases e o valor de pH 5,5 em água corresponde a $70 \%$ (Figura 3a). Na camada de $0-10 \mathrm{~cm}$, os valores de $\mathrm{pH}$ 5,5 em água e em $\mathrm{CaCl}_{2}$ correspondem a 70 e $77 \%$ de saturação por bases, respectivamente; para o $\mathrm{pH}$ 6,0 em água e em $\mathrm{CaCl}_{2}$, a $83 \%$ (Figura 3b e 3d). Na relação entre pH e saturação por bases, obtida por Quaggio (2000), na camada de 0-20 cm, em solos de São Paulo, o pH em água de 5,5, ou em $\mathrm{CaCl}_{2}$ de 5,0, corresponde à saturação por bases de $50 \%$ e o pH em água de 6,0, a $60 \%$.

A pequena diferença entre os valores de $\mathrm{pH}$ em água e em $\mathrm{CaCl}_{2}$ indica que a influência de sais foi
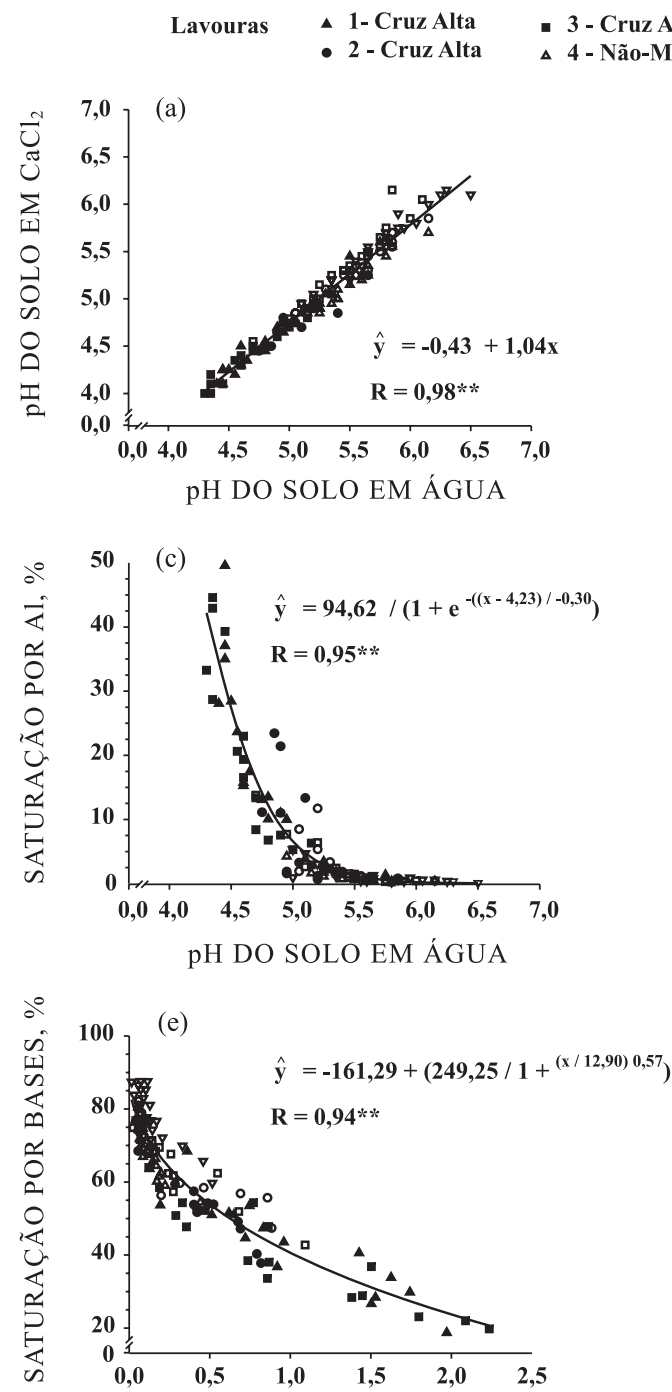

ALUMÍNIO TROCÁVEL, $\mathrm{cmol}_{\mathrm{c}} \mathrm{dm}^{-3}$
- 5 - Ibirubá $\quad \nabla 6$ - Ibirubá

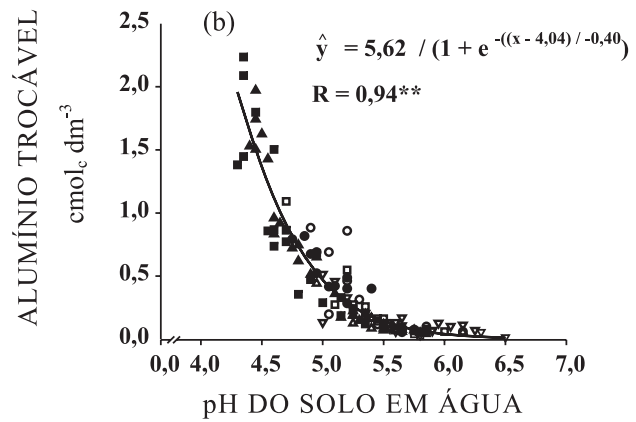

(d)
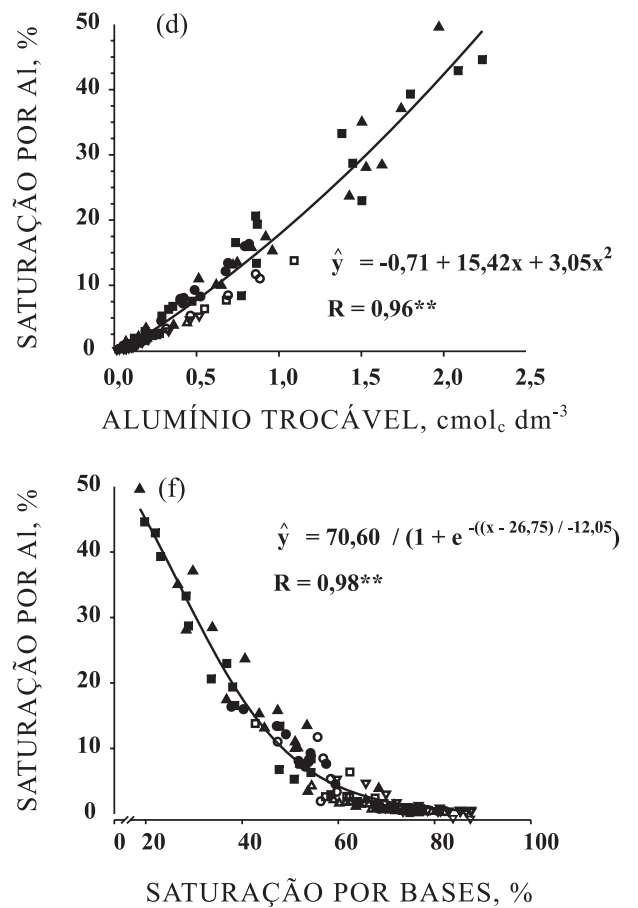

Figura 4. Relação entre o pH em água e o pH em $\mathrm{CaCl}_{2}$ (a), o alumínio trocável (b), a saturação por alumínio (c), entre o alumínio trocável e a saturação por alumínio (d), a saturação por bases (e) e entre a saturação por bases e a saturação por alumínio (f), em amostras de solo coletadas na camada de 0-20 cm de profundidade, em lavouras no SPD. 


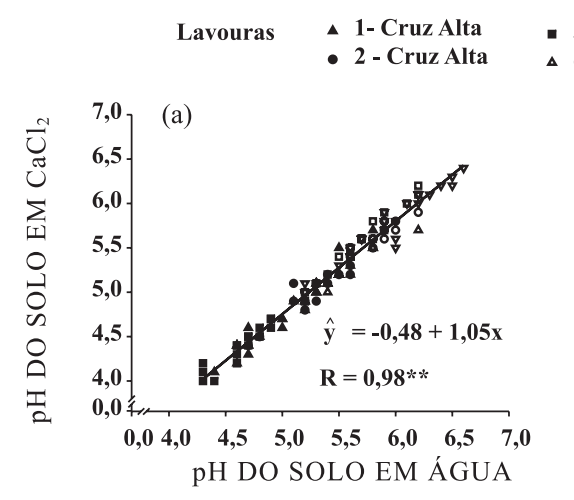

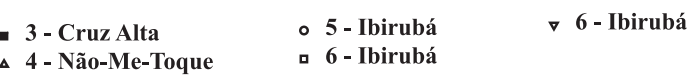
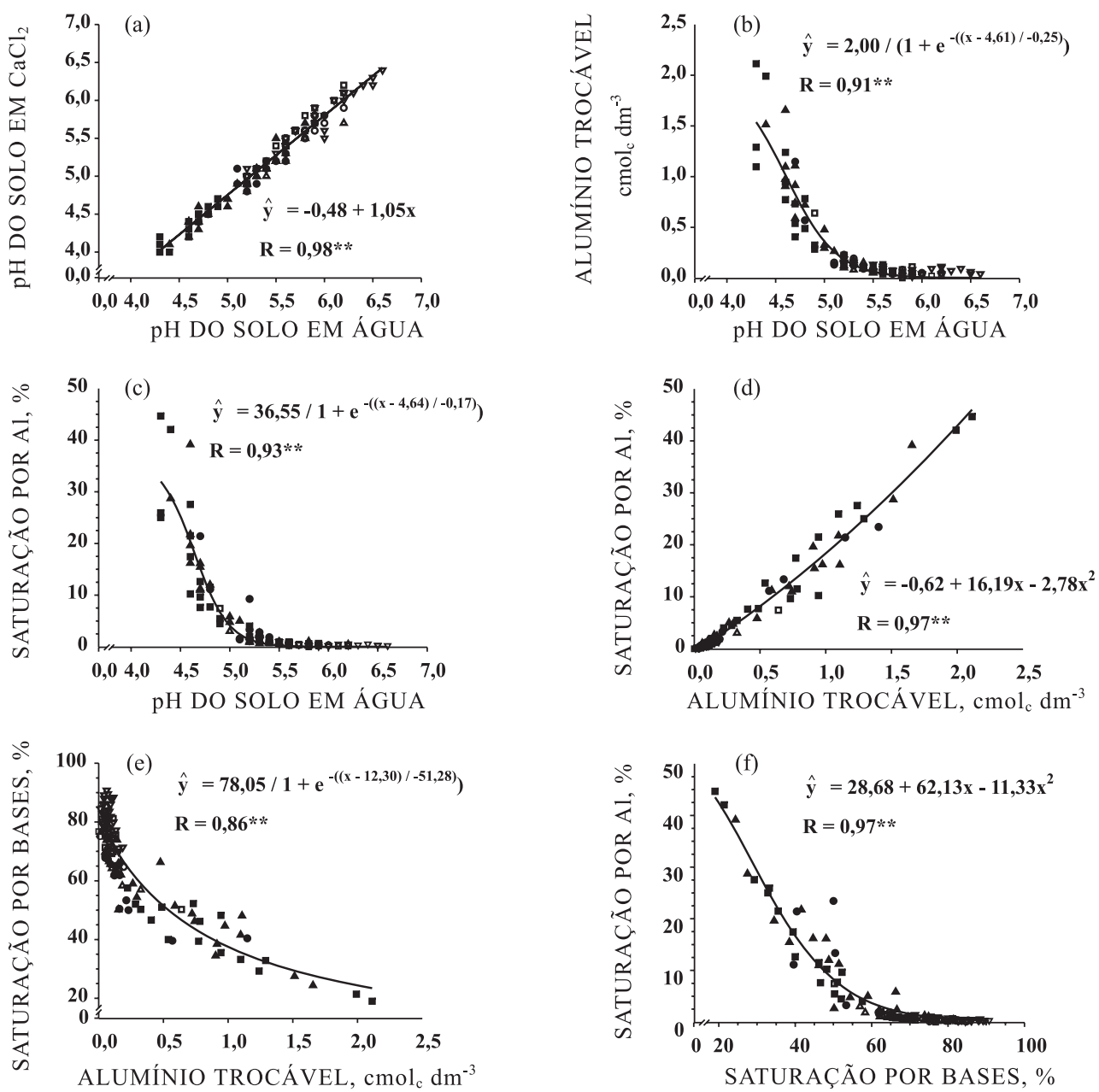

Figura 5. Relação entre o pH em água e o pH em $\mathrm{CaCl}_{2}(\mathrm{a})$, o alumínio trocável (b), a saturação por alumínio (c), entre o alumínio trocável e a saturação por alumínio (d), a saturação por bases (e) e entre a saturação por bases e a saturação por alumínio (f), em amostras de solo coletadas na camada de 0-10 cm de profundidade, em lavouras no SPD.

pouco importante nas áreas de lavouras no SPD (Figuras 4a e 5a). Em geral, a variação ocorrida neste trabalho foi de somente 0,2 unidade de $\mathrm{pH}$, para menos, na solução de $\mathrm{CaCl}_{2}$ na camada de $0-20 \mathrm{~cm}$ e de 0,3 unidade na camada de 0-10 cm. São valores semelhantes aos obtidos por Ciprandi (1993), de 0,3 a 0,4 unidade, para solos do Rio Grande do Sul no PC. Esses valores são, no entanto, menores do que a 0,6 unidade observada na camada de $0-20 \mathrm{~cm}$ em solos de São Paulo, também no PC (Raij, 1989; Quaggio, 2000). Tais diferenças entre os valores de $\mathrm{pH}$ em água e em $\mathrm{CaCl}_{2}$ devem-se, provavelmente, à maior capacidade de tamponamento de acidez dos solos do Rio Grande do Sul comparados aos solos de São Paulo.

O preparo do solo e a correção da acidez alteram a dinâmica do $\mathrm{Al}$. O Al trocável é o componente da acidez do solo que mais limita o rendimento das culturas, e o pH em água é o indicador mais freqüentemente utilizado para a recomendação de calagem no PC (Kaminski, 1989) e no SPD (CFSRS/SC, 1995). A presença de $\mathrm{Al}$ trocável foi baixa com $\mathrm{pH}$ em água 5,5 ou maior: $0,2 \mathrm{cmol}_{\mathrm{c}} \mathrm{dm}^{-3}$ na camada de $0-20 \mathrm{~cm} \mathrm{e}$ $0,1 \mathrm{cmol}_{\mathrm{c}} \mathrm{dm}^{-3}$ na camada de $0-10 \mathrm{~cm}$ (Figuras $4 \mathrm{~b}$ e 5b). É provável que Al trocável quantificado em valores de $\mathrm{pH}$ mais altos que 5,5 deva-se ao método utilizado, uma vez que este determinado também o H trocável da solução (Kaminski, 1974; Volkweiss, 1989). Assim, o valor de referência de 5,5 do $\mathrm{pH}$ em água poderia ser utilizado como critério de calagem, pois, acima desse $\mathrm{pH}$, o $\mathrm{Al}$ trocável não causa toxidez às plantas. No entanto, esse indicador pode não ser adequado quando seu valor for menor que 5,5 para representar a possível ação do Al trocável, conseqüentemente, para a recomendação de calagem (Figuras 4b e 5b). Como observado por vários autores (Quaggio, 2000) e no SPD (Salet, 1998; Kaminski \& Rheinheimer, 2000), no PC, 
com menor teor de MO (menor CTC), a saturação por Al aumenta, assim como a sua labilidade e seu efeito tóxico. No SPD, o acúmulo de resíduos vegetais na superfície do solo pode diminuir o pH; no entanto, mesmo com esta redução de $\mathrm{pH}$, pode não ocorrer aumento da toxidez de Al, como seria esperado, pois, neste caso, sua toxidez às plantas pode ser diminuída ou neutralizada (Salet, 1998). Assim, o efeito fitotóxico é mais dependente da sua saturação na CTC efetiva do que da sua própria concentração (Kaminski \& Rheinheimer, 2000).

A saturação por Al poderia ser utilizada como critério de recomendação de calagem, pois também engloba o efeito das bases retidas na CTC efetiva (Kaminski, 1989; Sumner, 1997; Pöttker \& Ben, 1998; Quaggio, 2000). Contudo, seu uso pode não ser prático, pois seria limitado a valores de $\mathrm{pH}$ e de saturação por bases até 5,5 e $60 \%$, respectivamente (Figuras 4c, 4f, 5c, 5f). Além disso, a magnitude das relações com a saturação por $\mathrm{Al}$ é muito pouco influenciada pela espessura da camada de solo avaliada. Por ter uma relação estreita com a saturação por bases, esta pode ser usada em substituição à saturação por $\mathrm{Al}$ com vantagem.

A camada de solo, para diagnóstico da acidez do solo no SPD, provavelmente, deveria ser menor do que a utilizada no PC $(0-20 \mathrm{~cm})$, uma vez que os efeitos desse sistema ocorrem em forma de gradientes, mais intensos a partir da superfície do solo. Um desses efeitos é o aumento dos teores de MO, fonte de superfícies reativas e de $\mathrm{H}$ trocável, que tanto pode ser constituinte da acidez potencial ou da acidez ativa, como pode interferir na acidez trocável diminuindo a atividade e a toxidez de $\mathrm{Al}$ às plantas. Por isso, poderse-ia esperar que a magnitude das relações entre acidez potencial e os indicadores de acidez se assemelhasse mais com as observadas no sistema convencional, quando avaliadas na camada de $0-20 \mathrm{~cm}$ no SPD, porém que fosse muito distinta, se avaliadas na camada de 0-10 cm, em que ocorrem mudanças mais intensas, principalmente em solos com alta fertilidade e alta adição de resíduos vegetais. No entanto, isso não ficou evidente nos solos cultivados no SPD avaliados no presente trabalho, e a alteração na magnitude das relações entre os tipos e os indicadores de acidez do solo (Figuras 1 a 5) com as camadas de solo avaliadas não foi significativa. A tendência das relações continuou a mesma; por exemplo, com o aumento do $\mathrm{pH}$, diminuiu o $\mathrm{Al}$ trocável e aumentou a saturação por bases e os valores obtidos foram semelhantes àqueles do PC para ambas as camadas avaliadas.

O objetivo da recomendação de calagem é reduzir a acidez do solo para que esta não limite o rendimento das plantas. Por isso, é mais conveniente utilizar maior número de indicadores que, em conjunto, representem melhor o funcionamento do sistema solo. Neste trabalho, há evidências de que podem ser utilizados simultaneamente, e com vantagem, os indicadores pH do solo, em água ou em solução de $\mathrm{CaCl}_{2}$, e a saturação por $\mathrm{Al}$ e por bases.

\section{CONCLUSÕES}

1. As tendências centrais das relações estudadas entre indicadores de acidez e a acidez potencial e entre os próprios indicadores de acidez foram semelhantes àquelas mostradas para solos no $\mathrm{PC}$. Eventualmente, para algumas relações, como no caso entre o pH e a saturação por bases, a magnitude foi diferente.

2. Para fins de recomendação de calagem, os resultados mostraram que, para o SPD na região estudada, o solo pode ser amostrado tanto na camada de 0-10 cm como na de 0-20 cm.

\section{LITERATURA CITADA}

ANGHINONI, I. \& SALET, R.L. Amostragem de solo e as recomendações de adubação e calagem para o sistema plantio direto. In: NUERNBERG, N.J., ed. Conceitos e fundamentos do sistema plantio direto. Lages, Núcleo Regional Sul/SBCS, 1998. p.27-52.

BAYER, C. \& MIELNICZUK, J. Características químicas do solo afetadas por métodos de preparo e sistemas de cultura. R. Bras. Ci. Solo, 21:105-112, 1997.

BOHNEN, H.; MEURER, E.J. \& BISSANI, C.A. Solos ácidos e solos afetados por sais. In: MEURER, E.J. Fundamentos de química do solo. Porto Alegre, Universidade Federal do Rio Grande do Sul, 2000. p.109-125.

CAIRES, E.F. Manejo da fertilidade do solo no sistema plantio direto: Experiências no Estado do Paraná. In: REUNIÃO BRASILEIRA DE FERTILIDADE DO SOLO E NUTRIÇÃO DE PLANTAS, FERTBIO, 25., Santa Maria, 2000. Anais. Santa Maria, Sociedade Brasileira Ciência do Solo, 2000. CD-ROM

CAIRES, E.F.; CHUEIRI, W.A.; MADRUGA, E.F. \& FIGUEIREDO, A. Alterações de características químicas do solo e resposta da soja ao calcário e gesso aplicados na superfície em sistema de cultivos sem preparo do solo. R. Bras. Ci. Solo, 22:27-34, 1998.

CARBALLO, A.M.C. Atributos químicos do solo afetados por métodos de preparo e sistemas de culturas. Porto Alegre, Universidade Federal do Rio Grande do Sul, 2004. 83p. (Tese de Mestrado)

CATTANI, R.A. \& GALLO, J.R. Avaliação da exigência em calcário dos solos do Estado de São Paulo mediante correlação entre o $\mathrm{pH}$ e a porcentagem de saturação por bases. R. Agric., 30:49-60, 1955.

CIPRANDI, M.A.O. Avaliação da metodologia de determinação da acidez ativa e potencial em solos do Rio Grande do Sul. Porto Alegre, Universidade Federal do Rio Grande do Sul, 1993. 90p. (Tese de Mestrado) 
COMISSÃO DE FERTILIDADE DO SOLO - CFSRS/SC Recomendações de adubação e de calagem para o Estado do Rio Grande do Sul e Santa Catarina. 3.ed. Passo Fundo, Sociedade Brasileira Ciência do Solo - Núcleo Regional Sul, Embrapa/CNPT, 1995. 224p.

D'AGOSTINI, L.R. Noção de fertilidade em solos: (Re)emergindo sistêmica. In: CONGRESSO BRASILEIRO DE CIÊNCIA DO SOLO, 31., 2007, Gramado. Palestras. Gramado, Sociedade Brasileira de Ciência do Solo, 2007. 6p.

FIORIN, J. E.; CANAL, I. N. \& CAMPOS, B.C. Fertilidade do solo. In: SILVA, M.T.B. A soja em rotação de culturas no sistema plantio direto. Cruz Alta, FUNDACEP/ FECOTRIGO, 1998. p.35-96.

KAMINSKI, J. Acidez do solo e a fisiologia das plantas. In: KAMINSKI, J.; VOLKWEISS, J. \& BECKER, F.C. SEMINÁRIO SOBRE CORRETIVOS DA ACIDEZ DO SOLO, 2., Santa Maria, 1989. Anais. Santa Maria, Universidade Federal de Santa Maria, 1989. p.39-61.

KAMINSKI, J. Fatores de acidez e necessidade de calcário em solos do Rio Grande do Sul. Porto Alegre, Universidade Federal do Rio Grande do Sul, 1974. 96p. (Tese de Mestrado)

KAMINSKI, J. \& RHEINHEIMER, D.S. A acidez do solo e a nutrição mineral das plantas. In: KAMINSKI, J., ed.. Uso de corretivos da acidez do solo no plantio direto. Pelotas, Sociedade Brasileira Ciência do Solo - Núcleo Regional Sul, 2000. p.21-40.

KAMINSKI, J. RHEINHEIMER, D.S.; BARTZ, H.R.; GATIBONI, L.C.; BISSANI, C.A. \& ESCOSTEGUY, P.A.V. Proposta de nova equação para determinação do valor de $\mathrm{H}+\mathrm{Al}$ pelo uso do índice SMP em solos do RS e de SC. In: REUNIÃO ANUAL DA REDE OFICIAL DE LABORATÓRIOS DE ANÁLISE DE SOLO E DE TECIDO VEGETAL DOS ESTADOS DO RIO GRANDE DO SUL E DE SANTA CATARINA, 33., Frederico Westphalen 2001. Anais. Frederico Westphalen, Sociedade Brasileira Ciência do Solo - Núcleo Regional Sul - ROLAS, 2001. p.21-26.

MIELNICZUK, J.; RHEINHEIMER, D.S. \& VEZZANI, F.M. Interações fertilidade e conservação do solo. In: REUNIÃO BRASILEIRA DE FERTILIDADE DO SOLO E NUTRIÇÃO DE PLANTAS, FERTBIO, 25., Santa Maria, 2000. Palestras. Santa Maria, Sociedade Brasileira Ciência do Solo, 2000. CD ROM.

MIYAZAWA, M.; PAVAN M.A. \& FRANCHINI, J.C. Moléculas orgânicas relacionadas à química e à fertilidade do solo. In: REUNIÃO BRASILEIRA DE FERTILIDADE DO SOLO E NUTRIÇÃO DE PLANTAS, FERTBIO, 25., Santa Maria, 2000. Palestras. Santa Maria, Sociedade Brasileira de Ciência do Solo, 2000. CD- ROM.

NICOLODI, M.; ANGHINONI, I. \& GIANELLO, C. Relações entre o $\mathrm{pH}$ do solo e os indicadores de fertilidade e o rendimento de soja em lavouras no sistema plantio direto. In: REUNIÃO SUL-BRASILEIRA DE CIÊNCIA DO SOLO, 5., Florianópolis, 2004. Anais. Florianópolis, Sociedade Brasileira Ciência do Solo - Núcleo Regional Sul, 2004. CD-ROM.

NOLLA, A. \& ANGHINONI, I. Critérios para calagem para a soja no sistema plantio direto consolidado. R. Bras. Ci. Solo, 30:475-483, 2006.
PÖTTKER. D. \& BEN, J.R. Calagem em solos sob plantio direto e em campos nativos do Rio Grande do Sul. In: NUERNBERG, N.J. Conceitos e fundamentos do sistema plantio direto. Lages, Sociedade Brasileira Ciência do Solo - Núcleo Regional Sul, 1998. p.77-92.

QUAGGIO, J.A. Acidez e calagem em solos tropicais. Campinas, Instituto Agronômico de Campinas, 2000. 111p.

RAIJ, B. van; CANTARELLA, H.; QUAGGIO, J.A. \& FURLANI, A.M.C. Recomendações de adubação e calagem no Estado de São Paulo. 2.ed. Campinas, Instituto Agronômico, 1996. 300p. (Boletim Técnico, 100)

RAIJ, B.van. Acidez e calagem. In: KAMINSKI, J.; VOLKWEISS, J. \& BECKER, F.C. SEMINÁRIO SOBRE CORRETIVOS DA ACIDEZ DO SOLO, Santa Maria, 1989. Anais. Santa Maria, Universidade Federal de Santa Maria, 1989. p.74-100.

RIBEIRO, A.C.; GUIMARÃES, P.T.G. \& ALVAREZ V., V.H. Recomendação para o uso de corretivos e fertilizantes em Minas Gerais. Viçosa, MG, CFS MG/UFV, 1999. 359p.

SÁ J.C. Manejo da fertilidade do solo no sistema plantio direto. In: SIQUEIRA, J.O. MOREIRA, F.M.S. \& LOPES, A.S., eds. Inter-relação fertilidade, biologia do solo e nutrição de plantas. Lavras, Sociedade Brasileira Ciência do Solo, 1999. p.267-319.

SALET, R.L. Toxidez de alumínio no sistema plantio direto. Porto Alegre, Universidade Federal do Rio Grande do Sul, 1998. 117p. (Tese de Doutorado)

SILVA, F.C. Manual de análises químicas de solos, plantas e fertilizantes. Brasília, Embrapa Solos, Embrapa Informática Agropecuária, Embrapa Comunicação para Transferência de Tecnologia, 1999. 370p.

SOUSA, D.M.G. \& LOBATO, E. Cerrado: Correção do solo e adubação. 2.ed. Planaltina, Embrapa Cerrados, 2004. 416p.

SOUSA, D.M.G.; MIRANDA, L.N.; LOBATO, E. \& CASTRO, L.H.R. Métodos para determinar as necessidades de calagem em solos dos Cerrados. R. Bras. Ci. Solo, 13:193198,1989

SUMNER, M.E. Procedures used for diagnosis and correction of soil acidity: A critical review. In: MONIZ, A.C.; FURLANI, A.M.C.; SCHAFFERT, R.E.; FAGERIA, N.K.; ROSOLEM, C.A. \& CANTARELLA, H., eds. Plant-Soil interactions at low $\mathrm{pH}$ : Sustainable agriculture and forestry production. Campinas, Brazilian Soil Science Society, 1997. p.195-204.

TEDESCO, M.J.; GIANELLO, C.; BISSANI, C.; BOHNEN,H. \& VOLKWEISS, S.J. Análise de solo, plantas e outros materiais. 2.ed. Porto Alegre, Universidade Federal do Rio Grande do Sul, 1995. 174p. (Boletim Técnico, 5)

VEZZANI, F.M. Qualidade do sistema solo na produção agrícola. Porto Alegre, Universidade Federal do Rio Grande do Sul, 2001. 184p. (Tese de Doutorado)

VOLKWEISS, S.J. Química da acidez dos solos. In: KAMINSKI, J.; VOLKWEISS, J. \& BECKER, F.C. SEMINÁRIO SOBRE CORRETIVOS DA ACIDEZ DO SOLO, 2., Santa Maria, 1989. Anais. Santa Maria, Universidade Federal de Santa Maria, 1989. p.7-38.

WIETHÖLTER, S. Calagem no Brasil. Passo Fundo, EmbrapaCNPT, 2000. 104p. 